Cómo citar este artículo en bibliografías / Referencia

P-Sande Vázquez-Sande, L García-Abad, P Pineda-Martínez (2019): “Comunicación interna y crisis reputacional. El caso de la Universidad Rey Juan Carlos”. Revista Latina de

Comunicación Social, pp. 1748 a 1760.

http://www.revistalatinacs.org/074paper/1408/91es.html

DOI: $10.4185 / R L C S-2019-1408$

\title{
Comunicación interna y crisis reputacional. El caso de la Universidad Rey Juan Carlos
}

\section{Internal communication and reputational crisis. The case of the Universidad Rey Juan Carlos}

\author{
Pablo-Vázquez-Sande [D] [ $\mathcal{G}$ ] Profesor Universidad de Santiago \\ de Compostela, USC, pablo.vazquez.sande@usc.es
}

Lito-García-Abad [D] [C] Profesor Centro de Estudios Superiores

Universitarios de Galicia, CESUGA, España-jlgarcia@usj.es

Paula-Pineda-Martínez [D] [CG]Profesora Universidad del País Vasco, UPV, España - paula.pineda@ehu.es

\begin{abstract}
s
[ES] La Universidad Rey Juan Carlos (URJC) se ha visto sacudida en los últimos meses por varios escándalos de corrupción que trascendieron a la opinión pública debido a la amplia cobertura que realizaron al respecto la mayoría de medios de comunicación españoles. En este contexto de crisis, se analizan los mensajes que en los últimos dos años (noviembre 2016 - octubre 2018) recibieron los estudiantes actuales de la institución por parte del rectorado de la URJC a través del correo electrónico corporativo. Se plantea una investigación que emplea como técnica el análisis de contenido y que se centra en un stakeholder especialmente sensible desde el punto de vista de la comunicación interna, los alumnos. Los resultados obtenidos reflejan tres tendencias: un comportamiento mayoritariamente reactivo y no proactivo; una demora considerable en la gestión de los diferentes conflictos que se sucedieron; y la preferencia por la estrategia de la transferencia de responsabilidades.
\end{abstract}

[EN] Introduction: In recent months, the Rey Juan Carlos University (URJC) has been shaken by a series of corruption scandals that became part of the public agenda due to extensive coverage carried out by most Spanish media organisations. Objectives: In this context of crisis, the focus of the research is the messages sent to students by the vice-chancellor's office via corporate email over the 
last two years (November 2016 - October 2018). Methods: To do this, content analysis is used as the main technique to approach the messages sent to the most crucial stakeholders, from the point of view of internal communication: the students. Results: Three trends have been identified: a mostly reactive, non-proactive behaviour; a considerable delay in the management of the different conflicts; and a preference for a blame redirection strategy.

\section{Keywords}

[ES] Comunicación de crisis; comunicación organizacional; comunicación interna; reputación; Universidad.

[EN] Crisis communication; corporate communication; internal communication; reputation; University.

Contents [ES] 1. Introducción. 1.1. El concepto de crisis. 1.2. Crisis en el sector público nacional español. 2. Metodología. 3. Resultados. 4. Discusión y conclusiones. 5. Referencia bibliográficas.

[EN] 1. Introduction. 1.1. The concept of crisis. 1.2. Crises in Spain's national public sector. 2. Methods. 3. Results. 4. Discussion and conclusions. 5. References.

Traducción de CA Martínez Arcos

(PhD, Universidad de Londres)

\section{Introducción}

\subsection{El concepto de crisis}

Aunque a menudo se habla de que vivimos en un mundo propenso a las crisis (Rodríguez-Toubes y Fraiz, 2011), no se trata tanto de un posible incremento cualitativo actual de las crisis producidas como del hecho cierto de que cualquiera de ellas es universalmente conocida por la desintermediación e instantaneidad de los canales de comunicación en manos de la población (Lorenzo Valdés, 2013; Sánchez Calero, 2001).

Desde el punto de vista teórico, según González Herrero (1998), una crisis se define como cualquier situación que corre el riesgo de: a) aumentar en intensidad, b) ser objeto de inspección por parte del gobierno y/o de los medios de información, c) interferir en el desarrollo normal de los negocios, d) poner en peligro la imagen positiva de que disfrutan la empresa o sus directivos y e) dañar el balance de resultados de la empresa de alguna forma.

Piñuel (1997) defiende que una crisis refleja el

"cambio repentino entre dos situaciones que pone en peligro la imagen y el equilibrio natural de una organización porque entre las dos situaciones (la situación anterior y la situación posterior a la crisis) se produce un acontecimiento súbito (inesperado o extraordinario) frente al cual una organización tiene que reaccionar comprometiendo su imagen y su equilibrio interno (como organización) y externo (como institución) ante sus públicos" (Piñuel, 1997: 167).

Además, la definición de crisis requiere siempre la existencia de un público que interprete que esa situación que se ha generado es difícil y hostil para sus intereses. Tal como sugiere Coombs (2007), 
el corpus de personas que conforman los públicos a los que se destinan las medidas de gestión y comunicación son los que vienen a percibir la amenaza que supone la crisis para la organización; de dicha percepción se pueden generar resultados, en ocasiones, negativos para los públicos interesados o stakeholders.

De este modo, la comunicación de crisis es aquella que se lleva a cabo frente a cualquier tipo de problema de la organización y que es necesario mitigar por medio de acciones comunicativas que consigan evitar que la variedad de públicos perciba una imagen de desprestigio de la entidad.

Realmente gestionar una situación de crisis de forma proactiva exige necesariamente estar preparado y entrenado para poner en marcha los procedimientos diseñados para ese escenario. Doctrinalmente se dice que la comunicación en esas situaciones funciona como el elemento cohesionador de una situación de caos, que permite de alguna forma darle sentido y orden a las múltiples variables que actúan en ese espacio temporal. Como afirma Guzmán Hennessey (2007), "para anticipar las crisis es necesario conocer a fondo la dinámica de la realidad; si uno descubre los patrones puede inferir, con algún grado de certeza, lo que ocurrirá". Esa es la base del Issues Management y de la definición de los estadios de pre-crisis, donde el seguimiento y la monitorización de asuntos potencialmente conflictivos permiten la construcción de escenarios probables sobre los que incidir para minimizar sus efectos e incluso hacerlos desaparecer. Y ahí entronca la disciplina con otras áreas y funciones organizacionales, como la gestión de los asuntos públicos o el lobbying (González Herrero, 2004).

La teoría del caos abre la puerta a escenarios en desorden donde quizá se puedan hallar formas alternativas de orden o, en las ideas de Heisenberg, la lógica de la incertidumbre. En ese nuevo orden donde cambios pequeños en origen pueden representar cambios sustanciales en destino o efectos, la gestión de los procesos de crisis intenta trasladar a situaciones de alta incertidumbre y complejidad escenarios de sistemas que buscan reducir la entropía generada. Así lo cree Habermas (Guzmán, 2007:143).

Para identificar si la organización está haciendo frente a una crisis o no, Medina (2017) considera que una situación de este tipo se puede definir a partir de la confluencia de una docena de variables: factor sorpresa, supone una amenaza, posee un carácter único, genera una desestabilización, acorta los tiempos de toma de decisiones, incrementa el nivel de tensión y estrés sobre todos los agentes implicados, se acentúa el rol de las emociones de los públicos, se incrementa la dificultad para el control de la conducta, se produce un desafío para la imagen y la reputación de la organización, la calidad de la información es con frecuencia escasa, aumenta la presión mediática sobre la organización y se acentúa el peligro para la posición de poder dentro de la corporación (Medina, 2017: 28-29).

Paralelamente, nunca debe perderse de vista en qué consiste el propio proceso de gestión de crisis, entendida por Fearn-Banks como

"proceso de planificación estratégica cuyo objetivo es anticipar situaciones de crisis y reaccionar adecuadamente durante una situación de crisis, o en una situación de acontecimientos negativos, un proceso que eliminar parte del riesgo y la incertidumbre y permite a la organización gestionar eficazmente su destino al mismo tiempo" (Fearn-Banks, 2001: 480). 
En este concepto se incluyen tanto la perspectiva comunicacional como la de las propias medidas abordadas para resolver la situación. A este respecto, los teóricos distinguen entre un modelo proactivo, que tiene lugar antes de que se active el riesgo y se produzca el conflicto, y otro reactivo, donde los esfuerzos se dirigen a contener su desarrollo. Cada uno de ellos supone la incorporación de una batería de medidas que cumplen con una función definida y delimitada (Marín, 2009:16).

De la importancia de la comunicación para la efectiva resolución de la crisis dan cuenta estudios como el de Losada y Zamora (2011), quienes muestran, partiendo del caso del Alakrana, cómo puede influir la gestión comunicativa de una crisis pública en el desenlace de la misma:

"Los distintos modos en los que la comunicación de un Gobierno puede contribuir no sólo a empeorar su propia imagen, sino también a generar un estado de ansiedad y preocupación e, incluso, a empeorar también el escenario futuro (...) lo que complica enormemente que se resuelva favorablemente" (Losada y Zamora, 2011:143).

En esta línea, si se centra la atención específicamente en el ámbito comunicacional, existen tres elementos que resultan cruciales de cara a la acertada gestión de la crisis: por una parte, el mensaje, que ha de ser "directo, comprensible y con la capacidad de aportar la máxima información a fin de reducir la sensación de angustia e incertidumbre entre sus públicos" (Medina, 2017: 63); el portavoz, que debe ser único y con reconocida solvencia, legitimidad y autoridad para hacer frente a los hechos; y el conocimiento óptimo de los públicos, ya que en contextos de crisis conviene

"alinearse adecuadamente con la información que manejan los públicos y sus expectativas. No podemos olvidar que los públicos se comprenden como parte responsable, directa o indirecta, de la resolución del conflicto y, por lo tanto, de una posible atenuación o cierre de la crisis" (Losada, 2010: 60).

En cuanto a las estrategias a seguir desde el punto de vista comunicativo una vez que ha estallado la crisis se encuentran las siguientes en opinión de teóricos como Gil (2013): en primer lugar, la del silencio, que consiste en no comentar los hechos y en "ignorar deliberadamente las acusaciones de las que se es objeto o bien, hablar lo menos posible de ellas" (Gil, 2013:36). Sin embargo, este tipo de reacciones se aconseja solo en casos de crisis leves o de bajo riesgo para la entidad.

Otra estrategia la constituye la negación, que debe reposar en la capacidad de demostrar la falsedad de los hechos imputados a la organización y, cuando una entidad opta por ella debe "negar en bloque los incidentes y rechazar cualquier interés que se le preste" (Gil, 2013:37).

En tercer lugar, la transferencia de responsabilidades es la que se elige cuando se acredita que existe una tercera parte que puede asumir la culpabilidad de la situación que se ha generado. Al igual que en la anterior, debe emplearse solo en aquellos contextos donde se pueda acreditar la veracidad de esa realidad. Teóricos como Gil dicen que, si el tercero que asume la culpa pertenece a la entidad, nos situaríamos ante una estrategia de 'cambio de fusibles', mientras que, si el tercero es ajeno a la organización, se denomina en el argot 'matar al mensajero'.

Por último, la de confesión incluye tanto el reconocimiento de la responsabilidad y la asunción de esta situación como las explicaciones que se otorgan.

\subsection{Crisis en el sector público nacional español}


En el sector público nacional se han llevado a cabo investigaciones recientes como la de Medina (2017) centrada en cuatro de las situaciones más controvertidas en este ámbito en el período 20102014 (cierre del espacio aéreo por la huelga de controladores, el terremoto de Lorca, el conflicto en el barrio burgalés del Gamonal y el contagio por el virus del Ébola). En su estudio, la autora defiende que las dimensiones que se deben tener en cuenta en un proceso de gestión de comunicación de crisis son seis: la propia crisis, la gestión de la crisis, la gestión de la comunicación, la gestión política, la relación con los medios y la propia evaluación. De entre ellas, defiende que la eficacia reposa, habitualmente, en los parámetros tres y cuatro (comunicación y política).

Un terreno especialmente prolífico para las investigaciones de este tipo es el de la sanidad, con propuestas como la de Nespereira García (2014) o la de García Frontiñán (2016), ambas sobre la Gripe A. A este respecto, el primer investigador se centra en las estrategias de construcción retórica del discurso, mientras que la segunda autora asegura que se trata de un episodio cuya gestión comunicativa pública se basó en la corrección, transparencia y disponibilidad de los gabinetes institucionales hacia los medios de comunicación.

Mientras, otras investigaciones del ámbito nacional avanzaron en el terreno de la comunicación online. Tal es el caso de Costa (2015), quien propone un estudio de caso monográfico sobre Bankia, o el de García Ponce (2013), centrada en el concepto de hostilidad en la red.

Paralelamente, otros estudios abordaron sectores dispares, desde el del terrorismo, con la propuesta de Zurutuza Muñoz (2009) sobre los atentados del 11-M, hasta el de la alimentación y las bebidas. Este último bloque lo configuran casos como el de de Enrique Jiménez (2007) con Fontaneda, respecto de la que muestra cómo la buena gestión de la crisis permitió a la marca potenciar su negocio; el de Vázquez y Fernández (2013) sobre el papel de la empresa de aguas de Mondariz durante la crisis de los pepinos en 2011; y también el de Saura Pérez (2003) con una aproximación panorámica al sector durante la última década de los 90.

Por último, investigaciones como la crisis de los astilleros de Cádiz (Goicoechea, 2015) ponen de relieve la importancia de monitorizar los "factores belígenos", aquellos indicadores que permiten predecir cambios en el entorno, y que entroncan directamente con una gestión proactiva de las crisis, donde tan importante es anticiparse a las mismas como reaccionar de forma acertada. Esto cierra perfectamente el círculo de la gestión de los conflictos potenciales de una organización: se trata de examinar y analizar las variables del entorno que pueden influir en la vida de una organización para adoptar las medidas más convenientes en cada momento y, a ser posible, incluso llegar a evitar el nacimiento de la crisis. En el peor de los escenarios, ese examen debe continuar tanto durante la crisis como al concluir esta, de forma circular y permanente en la gestión de los asuntos económicos, sociales, tecnológicos, políticos e internos que pueden influir en la organización. La necesaria postura activa de esta filosofía de gestión contrasta con la evidencia empírica del desinterés de las organizaciones por la previsión (Wilcox, 2001; Burgos y Pacheo, 2016).

\section{Metodología}

Esta investigación se centra en la Universidad Rey Juan Carlos que, desde noviembre de 2016, se ha visto salpicada por diversos casos que afectaron a su reputación y que se enmarcan en un contexto claro de crisis: las acusaciones de plagio a Fernando Suárez, rector de esta entidad entre 2013 y 2017; el supuesto fraude de la defensa del Trabajo Fin de Máster de la ex presidenta de la 
Comunidad de Madrid Cristina Cifuentes y del actual líder del principal partido de la oposición en España, Pablo Casado (ambos en el Instituto de Derecho Público dependiente de la URJC); los plagios en el Trabajo Fin de Máster que la ex ministra de Sanidad de España, Carmen Montón, defendió en esta universidad en el Máster Universitario en Estudios Interdisciplinares de Género; la investigación judicial sobre la obtención de 500 títulos de Derecho por parte de abogados italianos en la URJC; y la polémica en torno al convenio de colaboración suscrito en 2015, por iniciativa del actual rector, con la empresa European Airline Center SL para la explotación de un hangar que esta entidad privada usa aunque la universidad ha cedido terreno, ha costeado el millón de euros que ha supuesto la construcción y paga las facturas derivadas de su actividad.

Para abordar esta cuestión se ha diseñado una ficha de análisis propia con una veintena de ítems con los que se da respuesta a las siguientes preguntas de investigación. P1: ¿Se reconoce explícitamente que la organización vive una crisis? P2: ¿Cuál es la estrategia de reacción que predomina? P3: ¿Se detecta una gestión comunicativa y también sobre la propia causa de la crisis? P4: ¿Qué rasgos principales se advierten en la gestión comunicativa de la crisis?

El período analizado se extiende desde el 1 de noviembre de 2016, mes en que los medios comenzaron a difundir las acusaciones del plagio a Suárez, hasta el 31 de octubre de 2018, de modo que se aborda un período de dos años.

Este estudio se realiza desde la óptica de un público especialmente sensible a estos casos, como es la de los alumnos. Según las estadísticas de la propia Universidad, en el curso 2017-2018 rozaba los 46.000 matriculados entre estudiantes de grado (38.941), máster (5.975) y doctorado (950). A ellos habría que agregar los 2.539 alumnos de títulos propios y los 730 inscritos en la Universidad de Mayores, lo que eleva el total a 49.135 estudiantes.

Para ello, las técnicas que se han utilizado son la revisión bibliográfica y el análisis de contenido de un total de los 566 correos electrónicos que figuraban en la bandeja de entrada de un alumno del Grado de Publicidad y Relaciones Públicas. De ellos, se han seleccionado los 15 que de un modo específico aluden a alguna de las situaciones mencionadas al inicio de este apartado.

\section{Resultados}

El primer elemento relevante lo constituye el momento en que la URJC remitió los diferentes correos electrónicos a sus alumnos. Así, la primera reacción oficial de la entidad lle gó el 21 de diciembre de 2016 a las 15.29 horas, un mes más tarde de las primeras acusaciones de plagio a Fernando Ramos (las primeras informaciones datan del 16 de noviembre) y una semana después de que se produjese un comunicado conjunto de los rectores de otras universidades madrileñas en preocupación por dicha situación.

Cabe subrayar, además, que los estudiantes reciben esta información al día siguiente de que se hubiese anunciado a los medios de comunicación, contraviniendo de este modo el precepto de informar previamente a los públicos internos, una situación que se va a repetir en varias ocasiones a lo largo de todo el período estudiado.

Los alumnos no reciben más e-mails sobre asuntos vinculados a la crisis hasta el período comprendido entre el 7 y el 13 de febrero de 2017, cuando llegan a sus buzones contenidos de este 
tipo con motivo de la campaña electoral que vive la institución y que se había adelantado a raíz del plagio.

Por el contrario, a lo largo de 2018 se detecta un cambio de estrategia, con meses, como el de septiembre, incluso con tres correos electrónicos relativos a asuntos de crisis que vive la institución. En ese sentido, se observa que desde el 13 de abril de 2018 la Universidad muestra una tendencia mucho más proactiva, adoptando el hábito de trasladar a sus alumnos el informe que presenta el rector ante el consejo de gobierno una vez que se ha celebrado esta reunión.

En segundo lugar, otra cuestión singular se desprende de la fuente que envía dichos comunicados, ya que conviven cuatro remitentes: Vicerrectorado de Estudiantes (1), Junta Electoral Central (2, 3, 4, 5), Vicerrectorado de Comunicación (8) y, fundamentalmente, las cuentas vinculadas formalmente al rector. Al igual que en el aspecto anterior, también aquí se constata una evolución al centralizar el mensaje al máximo nivel de la entidad, en lugar de delegarlo en un vicerrectorado como ocurrió al inicio de la crisis.

En relación con esto, la amplia mayoría de comunicaciones se dirigen, de modo genérico, al conjunto de la comunidad universitaria, si bien al principio se aludía explícitamente y de un modo puntual a los estudiantes.

En tercer lugar, sobre el reconocimiento explícito de la situación de crisis por parte de la Universidad, se detecta que en el $46,7 \%$ de los casos esto no ocurre, ya que se alude a la situación de modo indirecto, con expresiones que evitan este término, como "situación injusta y desproporcionada" (1) o "los hechos acaecidos" (6). A este respecto, se observa que, hasta 2018, solo los correos remitidos por una de las candidatas a las elecciones (la rival del vencedor, considerado continuista con respecto al anterior rector) aludían a la crisis al hablar de una universidad "dañada en su reputación" (3), de la que hay que "extirpar las malas prácticas" (3).

Por el contrario, desde 2018 se admite con mayor frecuencia esta situación al aludir a hechos que "están afectando gravísimamente a la reputación y la imagen" (7), a la "crisis institucional que estamos viviendo" (8) o al "daño reputacional" (14).

En relación con todo ello, el reconocimiento de la gravedad de la coyuntura también sigue un camino paralelo, si bien predominan claramente aquellos correos electrónicos que admiten, con mayor o menor contundencia, la severidad de estos casos (73,3\%). Como ocurría anteriormente, en el primer comunicado no se admite esta situación, al igual que sucede en los emitidos en junio, julio y el primero de septiembre de 2018, donde, presumiblemente, la Universidad trata de alejar el foco mediático rebajando también el nivel de preocupación exterior que muestra. Sin embargo, la situación de la ex ministra de Sanidad devuelve el reconocimiento de la gravedad al primer plano.

Además, en la época electoral la consideración de la gravedad de los hechos oscila entre el posicionamiento tibio del candidato continuista, con expresiones como la de vivir un "momento difícil porque las circunstancias actuales me obligarán a ser muy transparente y a escuchar más a todos" (2) o "un tema que me preocupa, como a vosotros, es el prestigio de nuestra universidad" (5); y el posicionamiento mucho más contundente y combativo de la otra candidata: "una gravedad que no tiene en parangón en el ámbito universitario español y que pone en entredicho todo nuestro sistema científico" (3) y calificativos como "execrable", "agonía", "enferma", "impunidad" o "estructura clientelar y corrupta" con los que Berganza (4) define a la URJC. 
Fuera del contexto de la campaña electoral, el rector admite el 6 de abril de 2018 su "inquietud y pesadumbre", así como el "deterioro a la imagen" (6); mientras que una semana más tarde alude a su "enorme preocupación y tristeza por todos los hechos", entre los que incluye los "indicios de graves irregularidades administrativas" (7). Al mes siguiente, el rector admite que "sabemos que han existido comportamientos irregulares en nuestra institución" (9); mientras, al inicio del otoño de 2018, se compromete a "detectar y erradicar los comportamientos deshonestos y que tanto nos dañan" (13), una expresión similar a la que emplea el 26 de octubre de 2018, al calificar los hechos como aquellos que "tanto daño han hecho a nuestra institución" (15).

Con respecto a las alusiones explícitas a los hechos que han desencadenado la crisis, en el 33,3\% no se señalan de modo claro a qué se refiere la situación que vive la entidad. Así, resulta elocuente que en el correo electrónico que remite el Vicerrectorado de Estudiantes a los alumnos un mes más tarde de haber estallado el primer caso se aluda a "las noticias aparecidas recientemente en algunos medios de comunicación referidas al rector de nuestra Universidad, Fernando Suárez" (1) o a "esclarecer la situación de los estudios de Grado" (8).

Por el contrario, en otras situaciones sí se mencionan estos casos. En este sentido, son singularmente explícitos los correos electrónicos que remite la Junta Electoral General a petición de la candidata Rosa Berganza, quien habla de "escándalos que afectan gravemente a la ética profesional de quien la encabeza" (3) o de la "destrucción de nuestra institución” (3).

En relación con estas tres circunstancias (el reconocimiento explícito de la existencia de una crisis, el reconocimiento explícito de la gravedad de los hechos y la mención explícita del tipo de hechos que han desencadenado la situación), cabe subrayar que solo en el 33,3\% se adentran desde el punto de vista del contenido en las causas que han motivado los hechos que han conducido a la crisis. Uno de los casos lo constituye el correo electrónico de Berganza en que atribuye la situación a "aquellos que no se manifiestan de forma contundente contra el plagio académico" (4).

El grado de preocupación que muestra la URJC con respecto a esta situación también se advierte en que el 33,3\% de las comunicaciones que remitieron a los alumnos tuvieron un carácter monográfico, ya que en ellas solo se aludía a alguno o varios de los perfiles de la crisis institucional que atravesaba la entidad.

Asimismo, con frecuencia se señala el "compromiso de llegar hasta las últimas consecuencias" (7), que suele acompañarse de una orientación discursiva hacia el futuro, como por ejemplo al asegurar que "este Equipo de Gobierno se compromete a erradicar las malas prácticas pasadas y futuras que se pudieran dar en esta Universidad" (7) o que "se sigue trabajando en la redefinición de que estas cuestiones vuelvan a repetirse" (13).

Un cuarto elemento está relacionado con la gestión comunicativa que se realiza en relación al conjunto de medidas que se adoptan para restituir la situación anterior a la crisis. En este sentido, dos de cada tres comunicados inciden sobre las decisiones adoptadas para tratar de paliar el daño.

Dentro de ellas, oscilan las que son claramente de imagen sin entrar en el fondo del asunto, como adelantar la convocatoria de elecciones al cargo del rector (1), las genéricas en el marco de compromisos electorales, como impulsar un plan de regeneración (3) y aquellas que sí parecen reflejar una voluntad decidida por alcanzar las últimas consecuencias. 
En este último bloque se pueden incluir las "medidas que este equipo de gobierno va a llevar de forma contundente" (7), como la apertura de un expediente disciplinario, el control sobre el Instituto de Derecho Público o una auditoría; así como la implantación de un mecanismo de firma electrónica y doble autenticación para evitar fraude en actas, la presentación de una denuncia judicial y la redacción de un Código Ético (9); la creación del Vicerrectorado de Calidad, Ética y Buen Gobierno (11); la contratación de una auditoría forense (12); la apertura de un procedimiento de información reservada sobre el máster del Instituto de Derecho Público y el anuncio de una denuncia judicial si se percibe cualquier irregularidad (13); o la interposición de un recurso judicial (15).

Por otra parte, en quinto lugar, la estrategia que se ha seguido ha ido variando con el paso del tiempo. Así, frente a un primer mes en los que no se remitió a los estudiantes ningún comunicado al respecto de los plagios del rector (estrategia del silencio), después se impuso la de la transferencia de responsabilidades, que se detecta de modo evidente en un $60 \%$ de los correos que reciben los alumnos.

Con todo, casi siempre se trata de alusiones veladas al anterior equipo de gobierno del rectorado o de profesores implicados, de acuerdo con el relato del actual rector (6), que se afana en desligarse de todos los hechos. Así, se detectan expresiones del tipo "hechos acontecidos en el pasado de esta universidad y que están afectando en el presente" (7), "creo que es injusto que, por el comportamiento de unos pocos, se ponga en cuestión la labor de todos los miembros de esta institución" (9); "estoy convencido de que ni yo (que en ese momento no era rector) ni ningún órgano de la Universidad ha cometido la más mínima ilegalidad" (10); "respecto a errores que algunas personas de esta casa cometieron en el pasado" (13); "unos hechos del pasado de los que estamos teniendo que dar cuenta en el presente" (14); "solo así se podrán depurar las responsabilidades que, aunque residan en unos pocos, tanto, tantísimo daño nos han hecho" (15).

En otros casos, sobre todo en los mensajes electorales de Berganza, la estrategia de transferencia de responsabilidades supone una alusión mucho más directa y explícita que engloba tanto al rector Ramos como al candidato que aspira a sucederle:

"Nuestra universidad es objeto de atención pública, cuando no de oprobio, debido a los escándalos que afectan gravemente a la ética profesional de quien la encabeza actualmente y a la de quien aspira conmigo en estos comicios con una candidatura continuista" (3).

Paralelamente, es cierto que no se ha detectado ningún caso de una estrategia de negación, pero, como se señalaba anteriormente, el nivel de gravedad otorgado para estos hechos difirió notablemente según el momento.

Resulta llamativo que solo en el 6,7\% de los correos emitidos se halla la intención de pedir disculpas de modo explícito por la situación provocada. Sin embargo, estas disculpas se refieren no a los hechos que desencadenan la crisis sino a la gestión que hizo el rector de la URJC de este proceso:

"En todo momento he intentado esclarecer la verdad, podré haberme equivocado en decisiones puntuales o podré haber pecado por confiar en exceso en personas que no lo merecían (como quedó patente en mi anterior comparecencia del día 21 de marzo, por lo cual les pido disculpas públicamente), pero nunca he actuado de forma ilegal y no voy a permitir que nadie ensucie mi nombre ni el de la propia universidad" (6). 
Por otra parte, se detecta que, desde un punto de vista discursivo, la URJC trata de inculpar a los medios de comunicación en la situación crítica que atraviesa. Así, ya en el primer correo se señala que ese correo se envía a raíz de "las noticias aparecidas recientemente en algunos medios de comunicación referidas al rector de nuestra universidad, Fernando Suárez" (1).

Este tono se eleva considerablemente durante 2018, al expresar el actual rector "la sorpresa y frustración por el uso extensivo reciente de algunos titulares" (8) o también al apuntar que "la URJC está siendo cuestionada en algunos medios por la irresponsabilidad de algunos pocos" (13).

En una idea similar se incide al señalar que el archivo de las diligencias tras una denuncia presentada contra el rector

"aunque me afectaba a mí, directa y personalmente, es también una buena noticia para la institución, pues está en la senda de sacar a nuestra universidad del foco mediático negativo en el que nos hemos visto inmersos en los últimos meses" (12).

\section{Discusión y conclusiones}

El caso estudiado constituye un ejemplo de cómo una elevada presión mediática sobre una institución pública puede conducirla a un cambio radical en sus procedimientos de gestión de crisis hacia uno de sus stakeholders internos (los alumnos), con los que pasa de exhibir una actitud claramente reactiva en los momentos iniciales a mostrar un comportamiento más proactivo hacia el final del período estudiado.

De una estrategia fundamentada en el silencio se ha pasado a otra basada en la transferencia de responsabilidades (en algunas ocasiones más evidente y contundente que en otras), si bien casi nunca se piden disculpas por la situación generada. No se han detectado casos de negación de la crisis, aunque el grado de reconocimiento de este proceso, así como la preocupación mostrada sobre él, han ido incrementándose con el paso del tiempo, registrando un valle hacia el verano de 2018 y remontando a un nuevo pico desde septiembre de este año, en paralelo al descubrimiento de nuevas situaciones que afectan a una reputación ya de por sí dañada.

Por otra parte, sí se constata una estrategia de información directa a los alumnos sobre las medidas adoptadas para la restitución del daño causado. No obstante, esta comunicación en ocasiones llega una vez que ya los medios la han publicado, alterando la recomendación de suministrar primero contenidos a los públicos internos antes que a los externos.

Además, la existencia tanto de varios portavoces a la hora de afrontar estos quince procesos comunicativos como de estilos de mensaje en ocasiones contradictorios y en los que se inculpa a los medios a la hora de aludir la envergadura que ha alcanzado el caso entre la opinión pública parecen sugerir que la entidad no estaba preparada para afrontar una crisis de este calibre.

Por último, los resultados de esta investigación suponen una primera aproximación a este objeto de estudio, que se verá complementado en los próximos meses con una encuesta en la que una muestra de los casi 50.000 estudiantes de la URJC evaluarán la gestión comunicativa de la crisis que todavía afecta a su institución. 
- Los resultados de este artículo forman parte de las actividades de investigación promovidas a través de la Red XESCOM (R2014/026 XESCOM), apoyada por la Consellería de Cultura, Educación e Ordenación Universitaria de la Xunta de Galicia.

\section{Referencias bibliográficas}

JM Burgos Bravo \& C Pacheo Silva (2016): “Construcción de imaginarios en la gestión de la comunicación en situaciones de crisis en las organizaciones". En Revista de Estudios Cotidianos NESOP, 1 (4), junio, págs. 117 a 136.

WT Coombs (2007): “Attribution theory as a guide for post-crisis communication research", en Public Relations Review, 33 (2), páginas 135 a 139.

CV Costa (2015): Comunicación de crisis, redes sociales y reputación corporativa. Un estudio sobre el uso de las redes sociales como un discurso contrario a una campaña publicitaria (tesis doctoral). Madrid: Universidad Complutense. Recuperado el 5 de noviembre de 2018 de https://www.ucm.es/data/cont/media/www/pag-73273/TesisDoctoral_CarlosCosta-2015.pdf.

AM Enrique Jiménez (2007): La comunicación empresarial en situaciones de crisis. Estudio de caso: la crisis de Fontaneda (tesis doctoral). Barcelona: Universitat Autònoma. Recuperado el 11 de septiembre de 2018 de https://www.tdx.cat/bitstream/handle/10803/4142/aej1de1.pdf.

K Fearn-Banks (2001): “Crisis Communication: A Review of Some Best Practices”. En Heath, Robert L. (ed.), Handbook of Public Relations. Sage Publications Inc. California: Thousand Oaks.

E García Frontiñán (2016): Comunicación de crisis en los gabinetes de prensa sanitarios. Análisis de la producción periodística y de la información publicada sobre la Gripe A (tesis doctoral). Madrid: Universidad Complutense.

D García Ponce (2013): Comunicación de crisis y conflictos comunicativos 2.0. Hostilidad en la red (tesis doctoral). Málaga: Universidad de Málaga.

P Gil (2013): "El tratamiento informativo de la crisis y su gestión: Cómo salvar la situación”. En O Islas \& G Hernández: Investigando la comunicación en crisis. México: Razón y Palabra.

MM Goicoechea Margalef (2015): Herramientas de gestión de crisis en la comunicación institucional. Aplicación al análisis de la crisis de Astilleros de Cádiz 1995 (tesis doctoral). Sevilla: Universidad de Sevilla.

A González Herrero (1998): Marketing preventivo: la comunicación de crisis en la empresa. Barcelona: Bosch.

A González Herrero (2004): Issues Management: la gestión de asuntos potencialmente conflictivos. En M T Otero Alvarado (coord.). Actas del I Congreso Internacional de investigadores en Relaciones Públicas. Sevilla: Facultad de Comunicación de la Universidad de Sevilla, 17 y 18 de noviembre, Asociación de Investigadores en Relaciones Públicas (AIRP). Recuperado el 20 de noviembre de 2018 de https://idus.us.es/xmlui/handle/11441/38972 
A González-Herrero, A \& S Smith (2008): “Crisis communications management on the Web: How Internet-based technologies are changing the way public relations professionals handle business crises", en Journal of Contingencies and Crisis Management, 16 (3), págs. 143 a153.

M Guzmán Hennessey (2007): "La anticipación de las crisis una aplicación del enfoque del caos", en Eidos, $\mathrm{n}^{\circ} 7$, págs. 128 a 159.

M Lorenzo Valdés (2013): "Perspectivas de estudio en el manejo de crisis", en O Islas \& G Hernández (coord..), Investigando la comunicación en crisis, México: Razón y Palabra, págs. 81 a 95.

JC Losada Díaz \& R Zamora Medina (2011): “Gestión de la comunicación de crisis en secuestros: lecciones desde el 'Caso Alakrana”,, en Trípodos, 28. Barcelona: Universitat Ramón Llull, págs. 129 a 144; recuperado el 21 de septiembre de 2018 de https://www.raco.cat/index.php/Tripodos/article/view/247488.

JC Losada Díaz (2010): Comunicación en la gestión de crisis. Lecciones prácticas. Barcelona: UOC.

F Marín (2009): Comunicación de crisis. Madrid: LID Editorial.

RM Medina Mínguez (2017): Comunicación en situaciones de crisis: factores de éxito que favorecen su eficacia. Estudio comparado de casos de comunicación de crisis en el sector público español entre los años 2010-2014 (tesis doctoral). Murcia: Universidad de Murcia. Recuperado el 11 de octubre de 2018 de

https://www.tdx.cat/bitstream/handle/10803/405321/TRMMM.pdf?sequence=1\&isAllowed=y

J Nespereira García (2014): Estrategias discursivas en la comunicación de crisis sanitarias (retórica y teoría de la argumentación): el caso de la Gripe A en 2009. (tesis doctoral). Valladolid:

Universidad de Valladolid.

JL Piñuel Raigada (1997): Teoría de la comunicación y gestión de las organizaciones. Madrid: Síntesis.

D Rodríguez-Toubes Muñiz \& JA Fraiz Brea (2011): “Gestión proactiva de crisis en el turismo: una experiencia de estudio en Galicia”, en Pasos, 9 (2), págs. 279 a 289.

ML Sánchez Calero (2002): "Importancia de la Gestión de la Comunicación en periodos de crisis empresariales”, en Ámbitos 8 (7). $2^{\circ}$ semestre 2001 - 1er Semestre 2002 págs. 165 a 179.

MP Saura Pérez (2003): La gestión de la comunicación de crisis en el sector de alimentación y bebidas en España en el período 1990-2000. (tesis doctoral). Madrid: Universidad Complutense.

M Vázquez Gestal \& AB Fernández Souto (2013): "Las marcas en los contextos de crisis. La empresa aguas de Mondariz y su respuesta a la crisis de los pepinos", en Historia y Comunicación Social, 18 (1). Madrid: Universidad Complutense, págs. 207 a 218; recuperado el 12 de septiembre de 2018 de http://revistas.ucm.es/index.php/HICS/article/view/43961/41566. 
D Wilcox (2001): Relaciones Públicas: Estrategias y Tácticas. Madrid: Pearson.

C Zurutuza Muñoz (2009): Reformulación dramatística de la comunicación de crisis frente al terrorismo: aplicación metodológica a los atentados del 11 de marzo de 2004 en España (tesis doctoral). Pamplona: Universidad de Navarra.

Cómo citar este artículo en bibliografías / Referencia

J Vázquez-Sande, L García-Abad, P Pineda-Martínez (2019): “Comunicación interna y crisis reputacional. El caso de la Universidad Rey Juan Carlos". Revista Latina de Comunicación Social, pp. 1748 a 1760.

http://www.revistalatinacs.org/074paper/1408/91es.html

DOI: $\underline{\text { 10.4185/RLCS-2019-1408 }}$

- En el interior de un texto:

J Vázquez-Sande, L García-Abad, P Pineda-Martínez (2019: 1748 a 1760) ...

o

...J Vázquez-Sande et al, 2019 (1748 a 1760)

Artículo recibido el 20 de junio. Aceptado el 27 de septiembre Publicado el 6 de noviembre de 2019 